\title{
SENSITIVITY ANALYSIS IN OPTIMAL DESIGN OF COMPOSITE STRUCTURES WITH CURVILINEAR FIBRES
}

\author{
W. Szteleblak ${ }^{*}$
}

\begin{abstract}
Sensitivity analysis of composite structures with curvilinear fibres is considered in the paper. Fibres orientation and fibres volume are taken as design parameters. General functionals of kinematic fields are analyzed. Sensitivity formulas are expressed in terms of primary and adjoint states. Next, sensitivity coefficients are used in the incremental process of optimization. Numerical example illustrates applicability of the proposed method.
\end{abstract}

Keywords: composite structures, curved fibres, optimization, adjoint states, sensitivity analysis

\section{Introduction}

Structural design is concerned with an appropriate choice and determination of design parameters describing a structure. The structure has to fulfil constraints imposed on the admissible stress, displacement, stability as well as geometrical and technological limitations. However, the choice and determination of structural parameters is not unique. One can design many kinds of structures which fulfil imposed constrains but differ with respect to their weight or manufacturing cost. Thus, in order to obtain optimal or improved solution optimization procedures should be implemented.

In the current paper, the method of optimization of composite plates is presented. The hitherto existing literature on optimization of composite plates deals mostly with stacking sequence, fibre orientation or plies thickness optimization. But also optimization of composites with curve fibres (Muc and Ulatowska, 2010), optimization of variable thickness composites (Muc, 2017) or optimization of variable density composites (Pedersen, 2004), are gaining more and more popularity. Here, an attempt for simultaneous fibres volume and fibres orientation optimization is undertaken (Fig. 1).

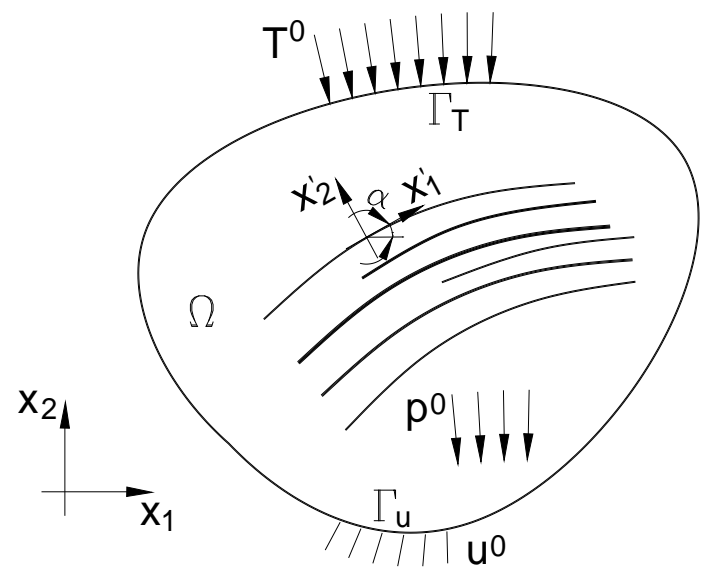

Fig. 1: Scheme of the plate.

Dr Wojciech Szteleblak, PhD.: Institute of Machine Design, Cracow University of Technology, Al. Jana Pawła II 37, 31-864 Cracow, Poland, wojciech.szteleblak@pk.edu.pl 


\section{Sensitivity analysis}

Consider now a plate occupying domain $\Omega \subset R^{2}$, with the boundary $\Gamma=\Gamma_{u}+\Gamma_{T}$ (Fig. 1). On $\Gamma_{T}$ tractions $\mathbf{T}^{0}$ are given and on $\Gamma_{u}$ displacement $\mathbf{u}^{0}$ are specified. Moreover, the plate is loaded in $\Omega$ by volume forces $\mathbf{p}^{0}$. Let us assume, that the following functional is defined in the plate domain

$$
G=\int_{\Omega} F(\boldsymbol{\varepsilon}, \mathbf{u}, \boldsymbol{\Psi}) d \Omega+\int_{\Gamma_{T}} g(\mathbf{u}) d \Gamma_{T}
$$

where $F$ is a function of strains $\boldsymbol{\varepsilon}$, displacements $\mathbf{u}$ and design parameters $\boldsymbol{\psi}$, while $g$ is a function of displacements $\mathbf{u}$. Vector of design parameters may contain topological, material, dimensional parameters, etc. But now, let us assume that the plate domain $\Omega$ is fixed, thus the shape of the plate does not undergo any changes. The first variation of the functional (1) with respect to material and topological parameters is expressed as follows

$$
\delta G=\int_{\Omega}\left(\frac{\partial F}{\partial \boldsymbol{\varepsilon}} \cdot \delta \boldsymbol{\varepsilon}+\frac{\partial F}{\partial \mathbf{u}} \cdot \delta \mathbf{u}+\frac{\partial F}{\partial \boldsymbol{\psi}} \cdot \delta \boldsymbol{\Psi}\right) d \Omega+\int_{\Gamma_{T}} \frac{\partial g}{\partial \mathbf{u}} \cdot \delta \mathbf{u} d \Gamma_{T} .
$$

Let us introduce the adjoint system of the following form (cf. Bojczuk and Szteleblak, 2008)

$$
\mathbf{N}^{a i}=\frac{\partial F}{\partial \boldsymbol{\varepsilon}}, \quad \mathbf{p}^{a 0}=\frac{\partial F}{\partial \mathbf{u}} \text { in } \Omega \quad \mathbf{T}^{a 0}=\frac{\partial g}{\partial \mathbf{u}} \text { on } \Gamma_{T}, \quad \mathbf{u}^{a 0}=\mathbf{0} \text { on } \Gamma_{u} .
$$

Now, the variation (2) becomes

$$
\delta G=\int_{\Omega}\left(\mathbf{N}^{a i} \cdot \delta \boldsymbol{\varepsilon}+\mathbf{p}^{a 0} \cdot \delta \mathbf{u}+\frac{\partial F}{\partial \boldsymbol{\psi}} \cdot \delta \boldsymbol{\psi}\right) d \Omega+\int_{\Gamma_{T}} \mathbf{T}^{a 0} \cdot \delta \mathbf{u} d \Gamma_{T} .
$$

In order to eliminate the unknown variations $\delta \mathbf{u}$ and $\delta \boldsymbol{\varepsilon}$, the principle of virtual work can be used

$$
\int_{\Omega} \mathbf{N}^{a r} \cdot \delta \boldsymbol{\varepsilon} d \Omega=\int_{\Omega} \mathbf{p}^{a 0} \cdot \delta \mathbf{u} d \Omega+\int_{\Gamma_{T}} \mathbf{T}^{a 0} \cdot \delta \mathbf{u} d \Gamma_{T},
$$

as well as the principle of complementary virtual work

$$
\int_{\Omega} \boldsymbol{\varepsilon}^{a} \cdot \delta \mathbf{N} d \Omega=\int_{\Gamma_{u}} \mathbf{u}^{a 0} \cdot \delta \mathbf{T} d \Gamma_{u}+\int_{\Gamma_{T}} \mathbf{u}^{a} \cdot \delta \mathbf{T}^{0} d \Gamma_{T}+\int_{\Omega} \mathbf{u}^{a} \cdot \delta \mathbf{p}^{0} d \Omega .
$$

Taking into account that variation of internal forces is as follows

$$
\delta \mathbf{N}=\delta(\mathbf{A} \boldsymbol{\varepsilon})=\delta \mathbf{A} \boldsymbol{\varepsilon}+\mathbf{A} \delta \boldsymbol{\varepsilon},
$$

and using homogenous boundary condition (3) 4 , the principle of complementary virtual work can be written in the form

$$
\int_{\Omega} \mathbf{N}^{a} \cdot \delta \boldsymbol{\varepsilon} d \Omega=-\int_{\Omega} \boldsymbol{\varepsilon}^{T} \cdot \delta \mathbf{A} \boldsymbol{\varepsilon}^{a} d \Omega+\int_{\Gamma_{T}} \mathbf{u}^{a} \cdot \delta \mathbf{T}^{0} d \Gamma_{T}+\int_{\Omega} \mathbf{u}^{a} \cdot \delta \mathbf{p}^{0} d \Omega .
$$

By an application of (5) and (8), the first variation of the functional (1) takes the following form

$$
\delta G=\int_{\Omega}\left(\frac{\partial F}{\partial \boldsymbol{\Psi}} \cdot \delta \boldsymbol{\Psi}-\boldsymbol{\varepsilon}^{T} \delta \mathbf{A} \boldsymbol{\varepsilon}^{a}+\mathbf{u}^{a} \cdot \delta \mathbf{p}^{0}\right) d \Omega+\int_{\Gamma_{T}} \mathbf{u}^{a} \cdot \delta \mathbf{T}^{0} d \Gamma_{T}
$$

If external load is fixed, it simplifies to

$$
\delta G=\int_{\Omega}\left(\frac{\partial F}{\partial \boldsymbol{\Psi}} \cdot \delta \boldsymbol{\Psi}-\boldsymbol{\varepsilon}^{T} \delta \mathbf{A} \boldsymbol{\varepsilon}^{a}\right) d \Omega .
$$

It often occurs that only some subdomain $\omega$ of a plate domain $\Omega$ undergoes changes, then the above variation is as follows

$$
\delta G=\int_{\omega}\left(\frac{\partial F}{\partial \boldsymbol{\psi}} \cdot \delta \boldsymbol{\Psi}-\boldsymbol{\varepsilon}^{T} \delta \mathbf{A} \boldsymbol{\varepsilon}^{a}\right) d \omega .
$$

When functional $G$ represents potential energy, we have

$$
\begin{aligned}
G & =\int_{\omega} \frac{1}{2} \mathbf{N} \cdot \boldsymbol{\varepsilon} d \omega-\int_{\omega} \mathbf{p}^{0} \cdot \mathbf{u} d \omega-\int_{\Gamma_{T}} \mathbf{T}^{0} \cdot \mathbf{u} d \Gamma_{T}= \\
& =\int_{\omega} \frac{1}{2} \boldsymbol{\varepsilon}^{T} \mathbf{A} \boldsymbol{\varepsilon} d \omega-\int_{\omega} \mathbf{p}^{0} \cdot \mathbf{u} d \omega-\int_{\Gamma_{T}} \mathbf{T}^{0} \cdot \mathbf{u} d \Gamma_{T},
\end{aligned}
$$

and the adjoint system has the following structure

$$
\mathbf{T}^{a 0}=-\mathbf{T}^{0}, \mathbf{p}^{a 0}=-\mathbf{p}^{0}, \mathbf{u}^{a 0}=\mathbf{0}, \mathbf{N}^{a i}=\mathbf{N}, \mathbf{N}^{a r}=-\mathbf{N},
$$




$$
\mathbf{N}^{a}=\varepsilon^{a}=\mathbf{u}^{a}=\mathbf{0} .
$$

In this case, variation of the functional $G$ becomes

$$
\delta G=\frac{1}{2} \int_{\omega} \boldsymbol{\varepsilon}^{T} \frac{\partial \mathbf{A}}{\partial \boldsymbol{\Psi}} \delta \boldsymbol{\psi} \boldsymbol{\varepsilon} d \omega
$$

As it is seen, this time no adjoint structure needs to be solved. In the next step, in order to calculate sensitivity of the potential energy, stiffness matrix A derivatives with respect to design parameters $\boldsymbol{\psi}$ should be introduced. Let us first write constitutive relation for composite layer of thickness $h$. It can be written as follows $\mathbf{N}=\mathbf{A} \boldsymbol{\varepsilon}$, but $\mathbf{A}=h \mathbf{Q}$ and hence

$$
\left[\begin{array}{l}
\sigma_{1} \\
\sigma_{2} \\
\sigma_{6}
\end{array}\right]=\left[\begin{array}{ccc}
Q_{11} & Q_{12} & 0 \\
Q_{21} & Q_{22} & 0 \\
0 & 0 & Q_{66}
\end{array}\right]\left[\begin{array}{l}
\varepsilon_{1} \\
\varepsilon_{2} \\
\varepsilon_{6}
\end{array}\right]
$$

Components of stiffness matrix, written in terms of composite material constants can be expressed as

$$
Q_{11}=\frac{E_{1}}{1-v_{12} v_{21}}, \quad Q_{22}=\frac{E_{2}}{1-v_{12} v_{21}}, \quad Q_{12}=Q_{21}=\frac{v_{21} E_{1}}{1-v_{12} v_{21}}, \quad Q_{66}=Q_{12} .
$$

Composite material constants can be calculated in the following way, involving material constants of fibre and matrix

$$
\begin{gathered}
E_{1}=E_{f} V_{f}+E_{m} V_{m}, \quad E_{2}=\frac{E_{f} E_{m}}{V_{m} E_{f}+V_{f} E_{m}}, \\
v_{12}=v_{f} V_{f}+v_{m} V_{m}, \quad v_{21}=\frac{E_{2}}{E_{1}} v_{12}, \quad G_{12}=\frac{G_{f} G_{m}}{V_{f} G_{m}+V_{m} G_{f}} .
\end{gathered}
$$

When rotated coordinate system is used, stiffness matrix must be transformed according to

$$
\overline{\mathbf{Q}}=\mathbf{T}^{\mathrm{T}} \mathbf{Q T},
$$

where

$$
\mathbf{T}=\left[\begin{array}{ccc}
\cos ^{2} \alpha & \sin ^{2} \alpha & \cos \alpha \sin \alpha \\
\sin ^{2} \alpha & \cos ^{2} \alpha & -\cos \alpha \sin \alpha \\
-2 \cos \alpha \sin \alpha & 2 \cos \alpha \sin \alpha & \cos ^{2} \alpha-\sin ^{2} \alpha
\end{array}\right] .
$$

Now, we must calculate derivations of stiffness matrix with respect to design variables, which in our case are specific fibre volume $V_{f}(\mathbf{x})$ and fibre orientation angle $\alpha(\mathbf{x}), \mathbf{x} \in \Omega$. Thus, the following variations of the potential energy should be specified

$$
\delta G_{v_{f}}=\frac{1}{2} \int_{\omega} \boldsymbol{\varepsilon}^{T} \frac{\partial \mathbf{A}}{\partial V_{f}} \delta V_{f} \boldsymbol{\varepsilon} d \omega, \quad \delta G_{\alpha}=\frac{1}{2} \int_{\omega} \boldsymbol{\varepsilon}^{T} \frac{\partial \mathbf{A}}{\partial \alpha} \delta \alpha \boldsymbol{\varepsilon} d \omega .
$$

The above derivations can be determined either analytically or numerically.

\section{Example}

The aim of the optimization process considered here is maximization of the global structure stiffness. The total potential energy is assumed as the measure of the global structural stiffness. The plate shown in Fig. 2a is analyzed. The plate is fixed on the left-hand edge and its thickness is $10 \mathrm{~mm}$. The material constants of fibres and matrix are as follows: $E_{f}=200 \mathrm{GPa}, v_{f}=0.3, E_{m}=20 \mathrm{GPa}, v_{m}=0.16$.
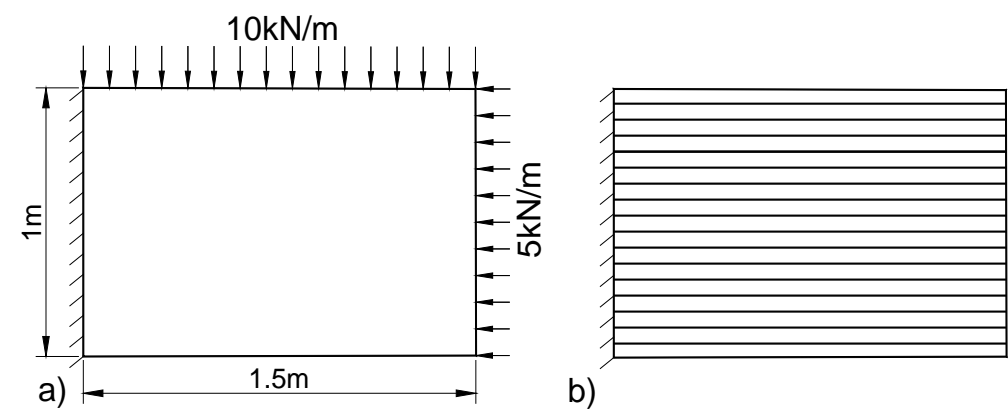

b)

Fig. 2: Static scheme of the plate. 
The problem of optimal design is defined in the following way

$$
\max _{V_{f} ; \alpha} G \quad \text { subject to } V_{f} \leq V_{f 0},
$$

where $G$ is the potential energy of the structure, $V_{f}$ stands for the specific fibre volume, $V_{f 0}$ is the maximum allowable specific fibre volume, which is 0.2 of the total plate volume, and finally $\alpha$ is the fibre orientation angle. The angle $\alpha$ is measured from the horizontal direction and is considered positive if fibre rotates counterclockwise. Initially, fibres are uniformly distributed and directed horizontally (Fig. 2b). The potential energy of the plate is $-1.68 \mathrm{~kJ}$. In order to solve the optimization problem, the plate is divided onto subdomains with constant design variables. Nine subdomains are chosen (Fig. 3a). Next, incremental procedure of gradient optimization is employed. In Fig.3b optimal fibres orientation and distribution are shown. Optimal values of design parameters are gathered in Tab. 1. The final potential energy increased to $-1.07 \mathrm{~kJ}$.

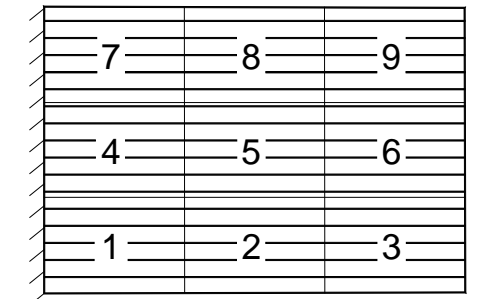

a)

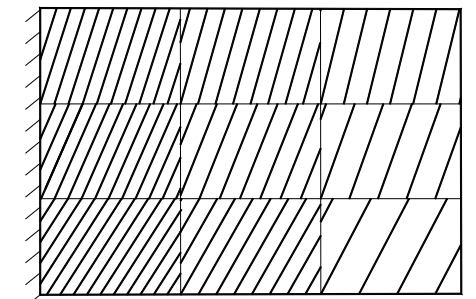

b)

Fig. 3: Initial and final design of the plate.

Tab. 1: Optimal values of design parameters.

\begin{tabular}{cccccccccc}
\hline Subdomain & 1 & 2 & 3 & 4 & 5 & 6 & 7 & 8 & 9 \\
\hline $\boldsymbol{V}_{\boldsymbol{f}}$ & 0.29 & 0.21 & 0.10 & 0.25 & 0.22 & 0.12 & 0.26 & 0.21 & 0.14 \\
\hline $\mathbf{a}$ & 57 & 60 & 62 & 65 & 68 & 70 & 74 & 75 & 76 \\
\hline
\end{tabular}

\section{Conclusions}

The expressions for sensitivity analysis of composite structures with respect to fibre volume and fibre orientation are derived in the paper. The obtained formulae can be used in optimization procedures. The formulated approach can also be used for other type of modification like thickness optimization. It can be also extended for topology and shape optimization.

\section{References}

Bojczuk, D. and Szteleblak, W. (2008) Optimization of layout and shape of stiffeners in 2D structures. Computers and Structures, 86, 13-14, pp. 1436-1446.

Hashin, Z. (1983) Analysis of composite materials- a survey. Journal of Applied Mechanics, 50, 3, pp. 481-505.

Mróz, Z. and Bojczuk, D. (2003) Finite topology variation in optimal design of structures. Structural and Multidisciplinary Optimization, 25, 3, pp. 153-173.

Muc, A. and Ulatowska, A. (2010) Design of plates with curved fibre format. Composite Structures, 92, 7, pp. 1728-1733.

Muc, A. (2018) Design of blended/tapered multilayered structures subjected to buckling constraints. Composite Structures, 186, pp. 256-266.

Parnas, L. and Suha, O. and Ceyhan U. (2003) Optimal design of composite structures with curved fibre courses. Composite Science and Technology, 63, 7, pp. 1071-1082.

Pedersen, P. (2004) Examples of density, orientation, and shape-optimal 2D-design for stiffness and/or strength with orthotropic materials. Structural and Multidisciplinary Optimization, 26, 1, pp. 37-49.

Szteleblak, W. and Bojczuk, D. (2005) Optimization of rib layout in plate structures, in: Proc. $16^{\text {th }}$ Int. Conf. on Computer Methods in Mechanics, Częstochowa, on CD-ROM. 